\title{
A Rare Case of Hypermobile Mesentery With Segmental Small Bowel Pneumatosis Cystoides Intestinalis
}

\author{
Chetan Rathi, Nirav Pipaliya, Prateik Poddar, Vikas Pandey, Meghraj Ingle, Prabha Sawant \\ Department of Gastroenterology, Lokmanya Tilak Municipal Medical College and General Hospital, Mumbai, India
}

Pneumatosis intestinalis is a rare condition that affects $0.03 \%$ of the population. Pneumatosis cystoides intestinalis (PCI) is characterized by the presence of multiple gas-filled cysts in the intestinal wall and the submucosa and/or intestinal subserosa. It is usually a secondary finding caused by a wide variety of underlying gastrointestinal or extragastrointestinal diseases. Here, we present the case of a 47-year-old man who was referred to our gastroenterology department with a history suggestive of intermittent small bowel obstruction associated with abdominal pain. Abdominal computed tomography demonstrated PCI of the small bowel. The mesentery and branches of the superior mesenteric artery and superior mesenteric vein were twisted with minimal pneumoperitoneum. Exploratory laparotomy was performed, and demonstrated segmental small bowel PCI secondary to hypermobile mesentery. The affected segment of the ileum was resected, and jejunoileal anastomosis was performed. Here, we report a rare case of segmental PCI probably due to repeated twisting of hypermobile mesentery. The clinical and imaging features of this disorder may mimic those of visceral perforation or bowel ischemia. PCI can be a cause of severe abdominal pain that may require surgical intervention. (Intest Res 2015;13:346-349)

Key Words: Mesenteric ischemia; Pneumatosis cystoides intestinalis; Pneumoperitoneum

\section{INTRODUCTION}

Pneumatosis cystoides intestinalis (PCI) was first described by Duo Vernoi during a cadaveric dissection in $1730 .{ }^{1}$ It is characterized by the presence of multiple gasfilled cysts in the intestinal wall and the submucosa and/ or gastrointestinal subserosa. The overall incidence of PCI in the general population has been reported to be $0.03 \%{ }^{2}$ It is usually a secondary finding caused by a wide variety of underlying gastrointestinal or extragastrointestinal diseases, but can be idiopathic in rare cases. ${ }^{3}$ Clinical signs and imaging features in these situations may mimic those of true abdominal viscera perforation, so correct diagnosis is imperative because the treatment of uncomplicated PCI

Received April 4, 2015. Revised April 27, 2015. Accepted April 27, 2015. Correspondence to Chetan Rathi, Department of Gastroenterology, Lokmanya Tilak Municipal Medical College and General Hospital, Sion, Mumbai 400022, India. Tel: +91-22-24063088, Fax: +91-22-24044154, E-mail: cdrathi@gmail.com

Financial support: None. Conflict of interest: None. is generally conservative. Management options range from supportive care to surgical resection. ${ }^{4}$ Here, we report a rare case of segmental PCI probably due to repeated twisting of hypermobile mesentery.

\section{CASE REPORT}

A 47-year-old man was referred to our gastroenterology department with recurrent episodes of abdominal pain over a period of 1 year associated with intermittent absolute constipation, abdominal distension, and occasional bilious vomiting. He had no history of any major surgery or medical illness. There was no history of fever, anorexia, or weight loss. His abdomen was distended but soft and non-tender with normal bowel sounds. Other systemic clinical examinations were normal. His routine blood parameters and liver and renal profiles were normal. Ultrasound examination of the abdomen and pelvis was normal. A CT scan of the abdomen suggested PCI in the small bowel loops and mesentery (Fig. 1). The mesentery and branches of the superior mesenteric

\footnotetext{
๑ Copyright 2015. Korean Association for the Study of Intestinal Diseases. All rights reserved.

This is an Open Access article distributed under the terms of the Creative Commons Attribution Non-Commercial License (http://creativecommons.org/licenses/by-nc/4.0)

which permits unrestricted non-commercial use, distribution, and reproduction in any medium, provided the original work is properly cited.
} 
artery and superior mesenteric vein were twisted (Fig. 2, 3) with minimal pneumoperitoneum (Fig. 4). A workup for connective tissue disorders was negative. An exploratory laparotomy was performed due to suspicion of bowel ischemia. Intraoperatively, the patient was found to have segmental PCI involving most of the ileum (Fig. 5). The small bowel mesentery was noted to be especially long and twisted. However, there was no evidence of bowel ischemia. The colonic loops and other intraperitoneal organs were entirely normal. The affected segment of the ileum was resected, and jejunoileal anastomosis was performed. The patient recovered postoperatively without complications. Histological examination of the resected specimen confirmed the diagnosis of PCI (Fig. 6).

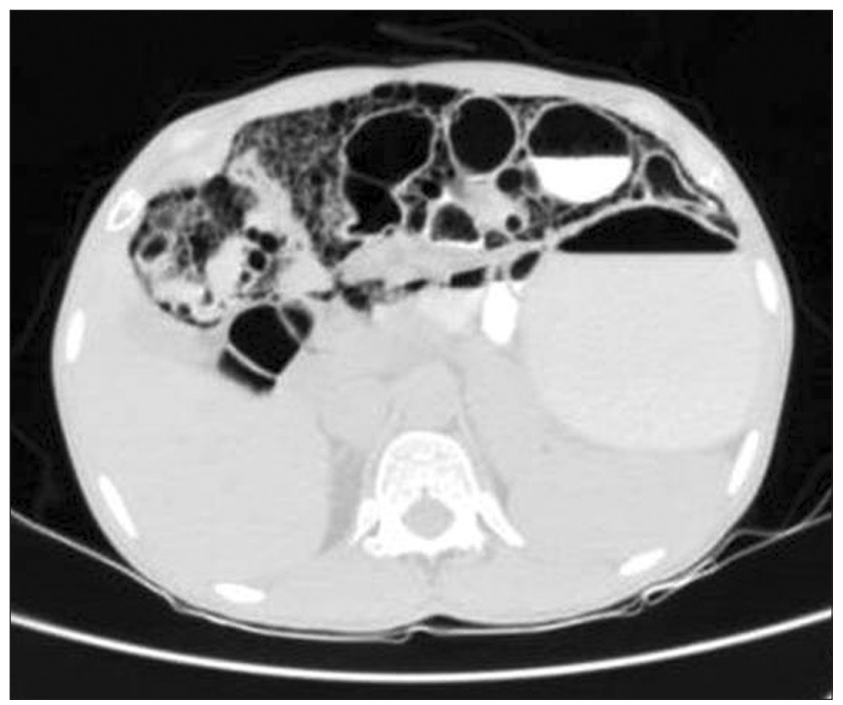

Fig. 1. CT findings. Contrast-enhanced CT of the abdomen (lung window) demonstrating pneumatosis cystoides intestinalis involving the small bowel loops.

\section{DISCUSSION}

PCI is a rare condition characterized by the presence of air-filled cysts in the bowel wall and mesentery. PCI may occur anywhere in the gastrointestinal tract. A previous study reported that $20 \%-51.6 \%$ of all PCI cases involve the small intestine, 36\%-78\% involve the colon, and $2 \%-22 \%$ include both the small intestine and the colon. When the air-filled cysts rupture they cause pneumoperitoneum, which is often benign. ${ }^{4}$

The pathogenesis of PCI has been extensively discussed, and multiple theories have been proposed. Three different possibilities for the origin of gas within the intestinal wall have been suggested: intraluminal gas, pulmonary gas, and gas produced by bacteria. Two basic mechanical features are responsible for the intrusion of intraluminal gas into the bowel wall: mucosal injury (the most important and prevalent feature) and increased intraluminal pressure, or a

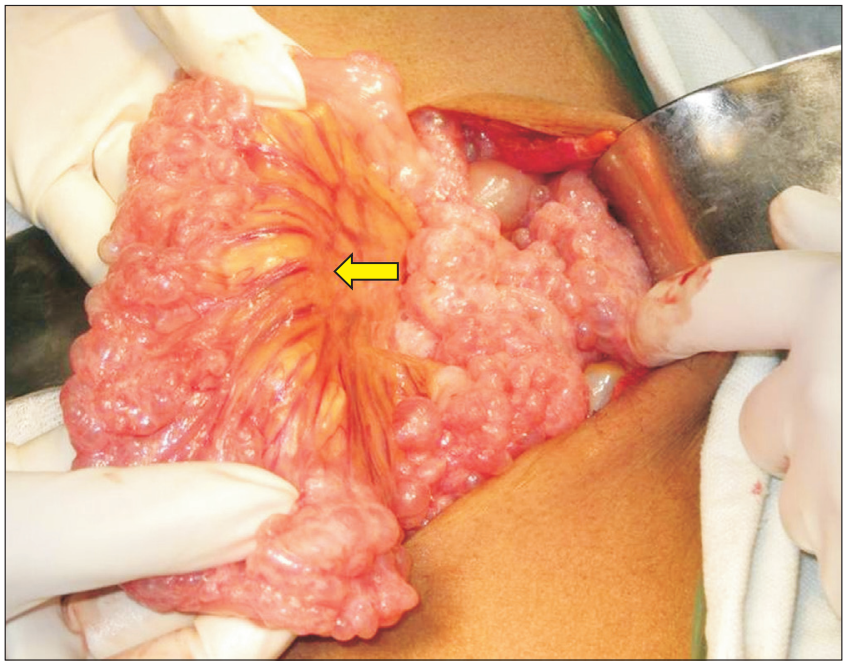

Fig. 3. Intra-operative findings. Segmental pneumatosis intestinalis involving the ileal loops. The small bowel mesentery is long and hypermobile (arrow).

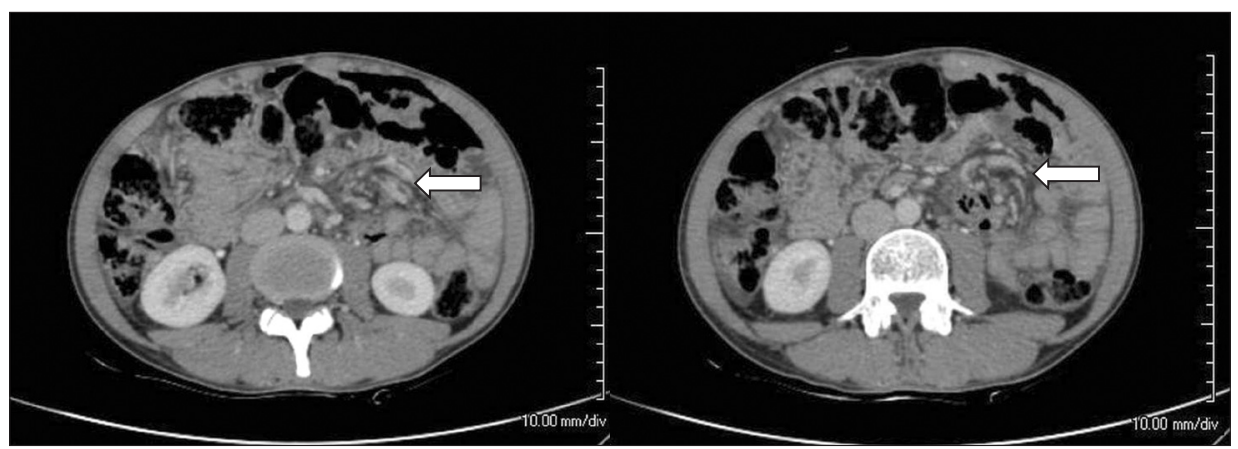

Fig. 2. CT findings. Contrast-enhanced CT of the abdomen (axial section) demonstrating twisting of the mesentery and branches of the superior mesenteric artery and vein (arrow). 


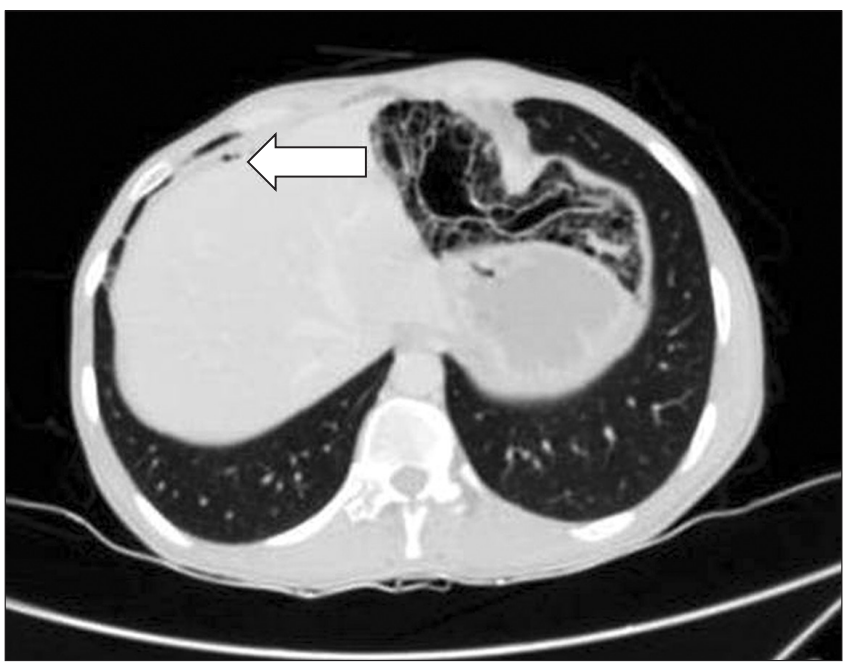

Fig. 4. CT findings. Contrast-enhanced CT of the abdomen (lung window) demonstrating a tiny focus of pneumoperitoneum in the perihepatic region (arrow).

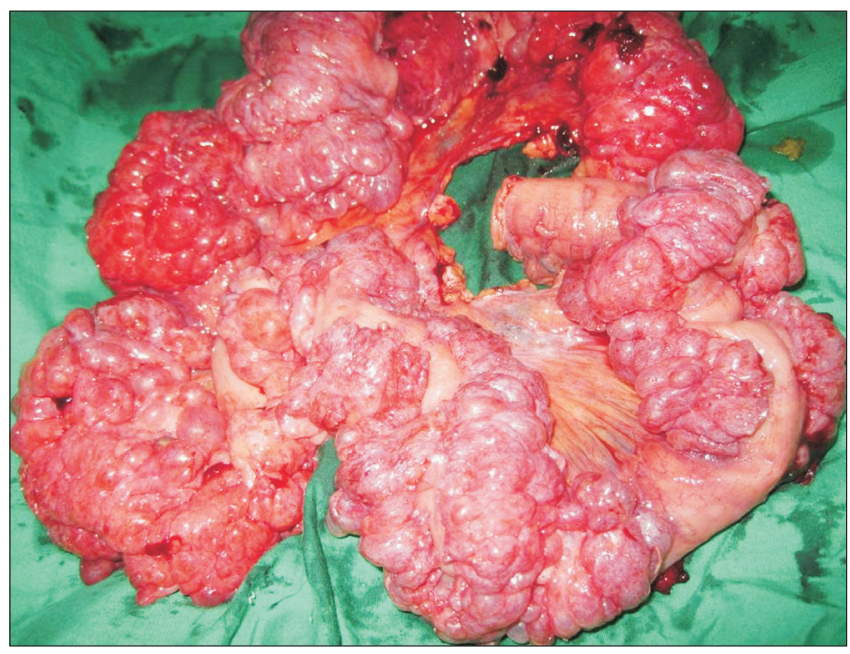

Fig. 5. Gross findings. Resected specimen of ileum with pneumatosis cystoides intestinalis.

combination of both. Increased intraluminal pressure may be produced by bowel obstruction, ileus, or iatrogenically by upper or lower gastrointestinal endoscopy. Damage to the mucosa may result from an inflammatory process, ischemia, a defect in the gut immune barrier, and steroid or cytotoxic medical therapy. ${ }^{5,6}$

PCI can be divided into primary and idiopathic (15\%) or secondary $(85 \%) .{ }^{3}$ Numerous gastrointestinal diseases, including necrotizing enterocolitis, $\mathrm{CD}$, UC, diverticular disease, and sigmoid volvulus may accompany PCI as a secondary cause. PCI has also been reported to be associated with some non-gastrointestinal diseases, such as chronic

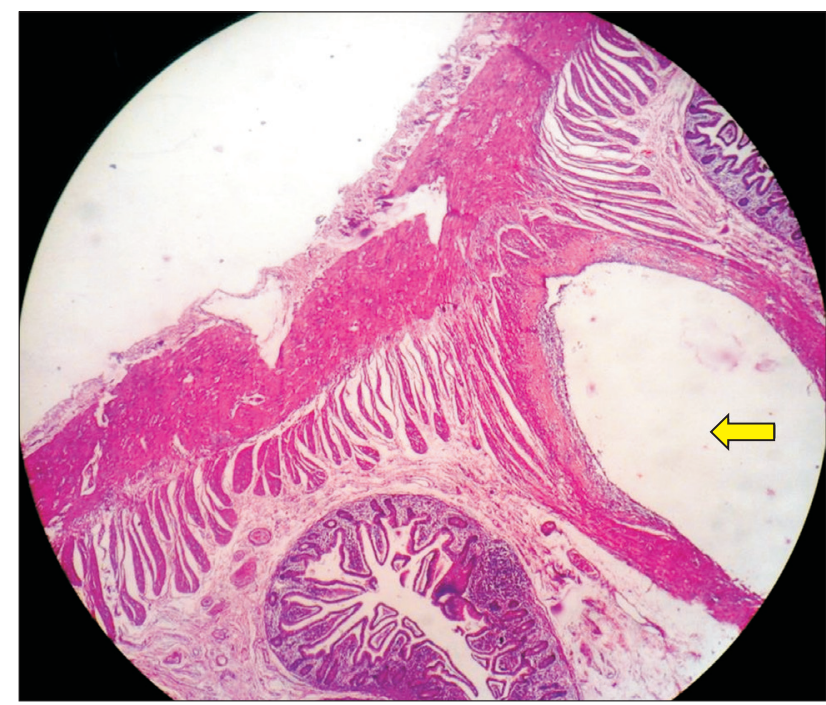

Fig. 6. Pathologic findings. Histopathology of the resected specimen of ileum demonstrating an air-filled cyst within the mucosa (arrow). Hematoxylin and eosin stain $(\mathrm{HCtE}, \times 40)$.

obstructive pulmonary disease and collagen tissue diseases. ${ }^{7}$ Disease location can act as a good predictor of etiology, with proximal disease usually being secondary to pyloric stenosis, gastric malignancy, or ulcers, and distal disease usually being a result of diverticulitis or mesenteric ischemia. ${ }^{8}$ Interestingly, segmental disease, as seen in our case, is rarely found. The PCI reported in the present case was probably secondary to repeated twisting of hypermobile mesentery resulting in ischemia with disruption of the mucosal barrier in the affected segment of bowel. Chronic obstructive pulmonary disease is often related to the development of pneumatosis intestinalis, but was excluded in our patient.

The overall incidence of PCI in the general population has been reported to be $0.03 \%$. $^{2}$ The disease is usually asymptomatic. Symptoms, if any, are usually secondary to an underlying disease. Patients may present with non-specific symptoms, such as abdominal discomfort, diarrhea, constipation, or rectal bleeding. Severe complications like volvulus, intestinal obstruction, bleeding, intussusception, and intestinal perforation may be seen in $3 \%$ of patients. ${ }^{9}$

The initial priority in the management of PCI is to clarify whether the underlying pathology is life threatening or benign. As seen in the present case, it is common for imaging studies such as plain radiography or CT to demonstrate features suggestive of free intraperitoneal gas (Fig. 4). It is important to consider the possibility of a perforated viscus, and thus crucial to correlate radiographic signs with clinical findings. Certain radiographic features, such as crescentic or linear gas collection, can be indicative of bowel infarction. ${ }^{10}$ 
In the case of a suspected life threatening condition such as bowel ischemia, surgical intervention in the form of an emergency exploratory laparotomy is mandatory. ${ }^{8}$ Conservative approaches, including nasogastric decompression, intestinal rest, antibiotic therapy, and oxygen are recommended for symptomatic patients with normal biochemical parameters who are confirmed by radiological examination to have no intestinal ischemia. ${ }^{9}$ However, with high associated mortality rates of between $22 \%-50 \%$, non-surgical management should be used with caution. ${ }^{4}$

Here, we report a rare case of segmental PCI probably due to repeated twisting of hypermobile mesentery. Clinical and imaging features may mimic those of visceral perforation or bowel ischemia. PCI can be a cause of severe abdominal pain, which may require surgical intervention.

\section{REFERENCES}

1. Braumann C, Menenakos C, Jacobi CA. Pneumatosis intestinalis - a pitfall for surgeons? Scand J Surg 2005;94:47-50.

2. Heng Y, Schuffler MD, Haggitt RC, Rohrmann CA. Pneumatosis intestinalis: a review. Am J Gastroenterol 1995;90:1747-1758.
3. Kim KM, Lee CH, Kim KA, Park CM. CT Colonography of pneumatosis cystoides intestinalis. Abdom Imaging 2007;32:602605.

4. Morris MS, Gee AC, Cho SD, et al. Management and outcome of pneumatosis intestinalis. Am J Surg 2008;195:679-683.

5. Schröpfer E, Meyer T. Surgical aspects of pneumatosis cystoides intestinalis: two case reports. Cases J 2009;2:6452.

6. Slesser AA, Patel PH, Das SC, Leahy A, Livingstone J, Riaz AA. A rare case of segmental small bowel pneumatosis intestinalis: A case report. Int J Surg Case Rep 2011;2:185-187.

7. Sakurai Y, Hikichi M, Isogaki J, et al. Pneumatosis cystoides intestinalis associated with massive free air mimicking perforated diffuse peritonitis. World J Gastroenterol 2008;14:6753-6756.

8. Donovan S, Cernigliaro J, Dawson N. Pneumatosis intestinalis: a case report and approach to management. Case Rep Med 2011;2011:571387.

9. Arikanoglu Z, Aygen E, Camci C, et al. Pneumatosis cystoides intestinalis: a single center experience. World J Gastroenterol 2012;18:453-457.

10. Soyer P, Martin-Grivaud S, Boudiaf M, et al. Linear or bubbly: a pictorial review of CT features of intestinal pneumatosis in adults. J Radiol 2008;89:1907-1920. 